This is a post-peer-review, pre-copyedit version of an article published in Journal of Low Temperature Physics. The final authenticated version is available online at: https://doi.org/10.1007/s10909-017-1837-4

\title{
Coulomb Blockade and Multiple Andreev Reflection in a Superconducting Single Electron Transistor
}

\author{
Thomas Lorenz • Susanne Sprenger • \\ Elke Scheer
}

Received: 07.09.2017 / Accepted: 27.11.2017

\begin{abstract}
In superconducting quantum point contacts multiple Andreev reflection (MAR), which describes the coherent transport of $m$ quasiparticles each carrying an electron charge with $m \geq 3$, sets in at voltage thresholds $e V=2 \Delta / m$. In single electron transistors (SETs) Coulomb blockade, however, suppresses the current at low voltage. The required voltage for charge transport increases with the square of the effective charge $\mathrm{eV} \propto(m e)^{2}$. Thus, studying the charge transport in all-superconducting single electron transistors (SSETs) sets these two phenomena into competition. In this article we present the fabrication as well as a measurement scheme and transport data for a SSET with one junction in which the transmission and thereby the MAR contributions can be continuously tuned. All regimes from weak to strong coupling are addressed. We extend the Orthodox theory by incorporating MAR processes to describe the observed data qualitatively. We detect a new transport process the nature of which is unclear at present. Furthermore, we observe a renormalization of the charging energy when approaching the strong coupling regime.
\end{abstract}

Keywords Superconductivity · Coulomb Blockade · Mesoscopic Transport • Break Junction · Multiple Andreev Reflection · Single Electron Tunneling • Single Electron Transistor

\section{Introduction}

A small metallic island weakly coupled to two leads via tunnel junctions forms a single electron transistor (SET). The transistor-like behaviour is due to the

Thomas Lorenz · Susanne Sprenger · Elke Scheer

University of Konstanz, Department of Physics, Universitätsstr. 10, D-78464 Konstanz

Tel.: +49-7531-88-4686

Fax: +49-7531-88-3090

E-mail: thomas.lorenz@uni-konstanz.de

Konstanzer Online-Publikations-System (KOPS)

URL: http://nbn-resolving.de/urn:nbn:de:bsz:352-2-1105nqrn1nih89 
Coulomb blockade of charge transport that can be modulated by a gate electrode. The Coulomb blockade occurs because a charging energy $E_{\mathrm{C}}=e^{2} / 2 C_{\Sigma}$ is required to charge the island of capacitance $C_{\Sigma}$ by one additional electron [1. To describe the current through a SET using the Orthodox theory (OT) 2, 3, the charge states of the island must be well defined, leading to the demand for low temperature $k_{B} T \ll E_{\mathrm{C}}$. Additionally the coupling through the tunnel junctions must be low enough to preserve the charge states, which can be expressed by a requirement on the junction resistances $R>R_{K}=e^{2} / h$. When reducing the resistance of the tunnel junctions below $R_{K}$, perturbation theory has to be considered to account for more charge states contributing to the current. Coulomb blockade itself has been confirmed experimentally for resistances much lower than $R_{K}$ [4, 5, 6]. Jezouin et al. [7] studied the zero bias CB oscillations in SETs, consisting of a metallic island contacted by quantum point contacts with variable conductance $G$ formed in an $2 \mathrm{D}$ electron gas. They found the disappearance of the CB oscillations when $G \geq 1 / R_{K}=h / e^{2}$ for any contact.

In the superconducting state two additional energy scales come into play: the quasiparticle gap $\Delta$ and the Josephson energy $E_{\mathrm{J}}=\hbar I_{\mathrm{C}} / 2 e$, which represents the coupling between superconducting island and superconducting leads. In the case of $\Delta>E_{\mathrm{C}} \gg E_{\mathrm{J}}$ the charge state of the island is well defined while it is easier to charge the island than to break a Cooper pair, hence the superconductivity is altered by charging effects. Thus the quasiparticle onset is shifted to bias voltages $\mathrm{eV} \geq 4 \Delta$. Plenty of different current features composed of Andreev reflection (AR), Cooper pair and quasiparticle transport are observed at lower bias voltages [8, 4, 9, 10]. So far, the interaction of multiple Andreev reflection (MAR) [11, 12 and CB in all superconducting devices was only studied theoretically 13, although MAR is well known in single atom contacts and diffusive wires. Experimental studies of AR or MAR and arbitrary charging effects have only been carried out in hybrid superconducting devices [14, 15, 16].

The probability $P$ for MAR transporting $m$ charges scales with the transmission $\tau$ of the participating transport channels $P \propto \tau^{m}$ [17. Oxide tunnel barriers typically utilized in SSETs have many conductance channels of very low transmission [11, 18, 19. In point contacts featuring only a small number of conductance channels with intermediate transmission, MAR becomes important. Especially break-junctions have proven to feature only a limited number of transport channels and show MAR of high order in the atomic sized contact regime 20 .

\section{Sample Preparation}

To investigate the interaction of $\mathrm{CB}$ and MAR we designed an SSET (cf. Fig. 1 a) with one static and one adjustable contact. The static contact is realized by an $\mathrm{Al} / \mathrm{AlO}_{\mathrm{x}} / \mathrm{Al}$ tunnel barrier (TB), while a mechanically controlled break junction (BJ) forms the adjustable contact. Using the BJ we can in situ 
tune the junction's coupling through all regimes, enabling us to characterize the sample in the Orthodox regime before adjusting the BJ to an atomic size contact.
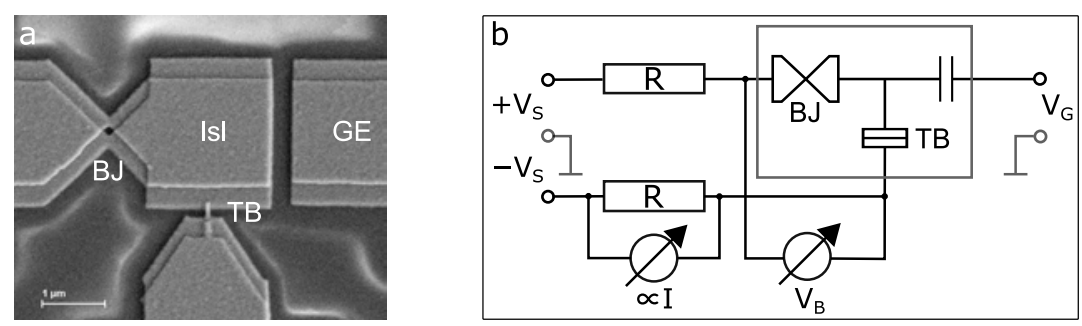

Fig. 1 SEM image (a) and bias scheme of the SSET. A break junction (BJ) and an oxide tunnel barrier (TB) define the island (Isl) to which the gate electrode (GE) couples capacitively. Two reference resistors $(\mathrm{R})$ of equal value in series with the sample preserve the symmetry of the voltage source $V_{\mathrm{S}}$. One of them is used to determine the current (I) through the sample. The bias voltage $V_{\mathrm{B}}$ is measured directly. The gate voltage $\left(V_{\mathrm{G}}\right)$ is applied against the common voltage of the bias.

We use shadow evaporation to fabricate the structure on a bendable bronze wafer covered by an insulating polyimide sacrificial layer, prepared as described in Ref. 21. We apply electron beam lithography to pattern the $2 \times 2 \mu^{2}$ island (Isl). The gate electrode (GE) is separated from the island by $200 \mathrm{~nm}$. The constriction on the left constitutes the BJ while a finger protruding from the other electrode forms the tunnel barrier (TB). We produce the tunnel barrier by first depositing $45 \mathrm{~nm}$ of aluminium followed by an oxidation in 0.1 mbar $\mathrm{O}_{2}$ for $30 \mathrm{~min}$. Evaporating a second $60 \mathrm{~nm}$ thick layer of aluminium under a different angle leads to an overlap of roughly $50 \times 50 \mathrm{~nm}^{2}$ and tunnel resistances of $25-125 \mathrm{k} \Omega$. The tunnel resistance typically increases by $10-15 \%$ when cooled down. The sample fabrication is finalized by a reactive ion etching process in oxygen to suspend the BJ.

In order to rule out an influence of the double constriction caused by the shadow evaporation, we performed several measurements on test samples featuring the same type of double constriction. The conductance histograms while opening and closing the double BJ show no deviation from the ones obtained using a single aluminium BJ [22]. We argue that the different layer thickness of the replicated BJs causes one of the junctions to break way before the second one is adjusted to the atomic point contact regime.

\section{Transport Measurements}

A symmetric four-point configuration provides the basis for the electrical measurement (cf. Fig. 1b). Two $100 \mathrm{k} \Omega$ shunt resistors, one of which is used for current measurement, are placed nearby in series with the sample at low temperatures. The bias voltage $\left(V_{\mathrm{B}}\right)$ is measured independently. This allows us to 
record the highly nonlinear current voltage (IV) characteristics with sufficient resolution even though the overall resistance of our sample changes in a wide range during the whole measurements. All measurement and bias lines are realized as twisted pairs. Signal and bias filtering is provided by capillary cables from 4 Kelvin to base temperature, copper powder 23 and RC low pass filters at different temperatures. A two-stage amplification scheme for both current and voltage with a fixed-gain preceding a variable-gain amplifier provides the adjustability for the different ranges. The gate voltage $\left(V_{\mathrm{G}}\right)$ of the SSET is applied against the common ground of the bias. A superconducting magnet offers the possibility to suppress superconductivity if desired. Transport measurements comprise recording IV characteristics by applying a slowly varying source voltage $\left(V_{\mathrm{S}}\right)$ for different constant gate voltages. We further process the data and generate the shown bias-gate-current maps by using statistical data binning and applying a Savitzky-Golay filter to smoothen the data and calculate the derivative numerically when required.

\section{Results and Analysis}

We present data from one sample tuned to different transport regimes recorded in the normal and superconducting state. Using the low temperature IV characteristics of the pristine sample, which showed no $\mathrm{CB}$ or dependence on the gate voltage, we determined the superconducting gap $\Delta=190 \mu \mathrm{eV}$ as well as the normal conducting resistance of the oxide tunnel barrier $R_{\mathrm{TB}}=112 \mathrm{k} \Omega$. Following this primary characterization, the sample was broken at $2.5 \mathrm{~K}$ to reduce the possibility of contamination in the BJ. For all measurements presented, the sample was cooled down to $T \leq 50 \mathrm{mK}$ after adjusting the BJ. The adjusted contact has a high time stability of more than $24 \mathrm{~h}$. This is achieved by using a stepper motor for driving the mechanics and the pronounced reduction ratio of the BJ. The time stability is required to measure the very same contact configuration in normal and superconducting state.

In order to assign the current features through the SSET to particular charge transport processes, two methods of evaluating the data are used. The first method is based on identifying the contributions to the current features using simple energy considerations while the second one involves simulating the current.

\subsection{Superconducting Transport Diagram}

In contrast to the normal conducting SET, where single electron tunnelling defines the shape of the Coulomb blockade, in the superconducting state more charge transport processes come into play. These processes include the coherent superconducting transport (i.e. transport of a Cooper pair or Josephson transport) and the dissipative transport of quasiparticle tunnelling, Andreev reflection (AR) and multiple Andreev reflection transferring three charges 
(from now referred to as MAR). Since the transport processes show a characteristic bias dependence (caused by the different number of charges transported $m$ and quasiparticles created $q$ ) these dependences can be utilized for identifying the transport mechanism.

In the Orthodox regime the number of electrons $n$ is a sufficient quantum number and the system's Coulomb energy $U(n)=(Q-n e)^{2} / 2 C_{\Sigma}$ is well defined, $Q=-C_{\mathrm{G}} V_{\mathrm{G}}+Q_{0}$ being the charge induced by the gate $\left(-C_{\mathrm{G}} V_{\mathrm{G}}\right)$ and the grounded substrate $\left(Q_{0}\right)$. Following Ref. [10], for positive bias $V_{\mathrm{B}}>0$, only charging the island through the TB and discharging the island through the BJ need to be considered. The fraction of the bias available for the transport over each junction $\left(\kappa_{\mp} V_{\mathrm{B}}\right)$ can be calculated using the simple model of a capacity voltage divider and depends on the difference of the junction capacitances $\delta C=C_{\mathrm{BJ}}-C_{\mathrm{TB}}$ solely. We find $2 \kappa_{\mp}=1 \mp \delta C / C_{\Sigma}$ where the $\kappa_{-}$is associated with transport over the BJ. For a given transport mechanism this bias voltage needs to provide the energy to change the islands charge $(U(n \mp m)-U(n))$ and create quasiparticles $(q \Delta)$.

$$
\begin{aligned}
m e \kappa V_{\mathrm{B}} & =(U(n \mp m)-U(n))+q \Delta \\
& =2 m E_{\mathrm{C}}\left[\left(\frac{C_{\mathrm{G}} V_{\mathrm{G}}-Q_{0}}{e}+n\right)+\frac{m}{2}\right]+q \Delta
\end{aligned}
$$

This equation gives the threshold bias voltage for the dissipative transports of quasiparticle tunnelling $(m=1, q=2)$, the $\operatorname{AR}(m=2, q=2)$ and the MAR ( $m=3, q=2$ ). Coherent Cooper pair transport is possible when the resonance condition $(m=2, q=0)$ is met. These thresholds and the resonance condition evaluated for different island charge states $n$ make up a family of lines in the bias/gate-voltage plane. This transport diagram, which solely depends on the parameters $E_{\mathrm{C}}$ (or $C_{\Sigma}$ ), $\delta C, C_{G}, \Delta$ and $Q_{0}$, helps identifying the transport processes involved in the observed current features. While negative slopes indicate a transport over the BJ, positive slopes correspond to transport over the TB.

Observing a current through the SSET requires transport through both junctions. Therefore, the number of electrons on the island $n$ needs to cycle through different charge states. By combining the different superconducting transport processes, many cycles are possible. The simplest cycle is made up of quasiparticle transport through both junctions involving the charge states $n$ and $n \pm 1$, equivalent to the situation in a non-superconducting SET. We will refer to this cycle as e-e cycle or quasiparticle onset.

The most prominent sub-gap feature is the so called J-QP cycle [8, 24, featuring a Josephson transport through one of the junctions and the transport of two quasiparticles through the other junction. As Josephson transport requires resonance, this cycle only contributes where the condition is met, thus leading to a peak in the current. The island charge passes through $n \Rightarrow n \pm 1 \Rightarrow n$ as for non-zero bias the resonant Josephson transport needs some coherent dissipative transport over the other junction to happen. Therefore, in the JQP cycle Josephson and quasiparticle transport combine into J-e transport. 
Another $n \Rightarrow n \pm 1 \Rightarrow n$ cycle consists of two alternating J-e transports with the resonance met at both junctions consecutively. This process is known as $3 \mathrm{e}$ [8] and shows the highest contribution near the intersection of two Josephson lines.

Taking into account the other dissipative transport processes of (M)AR, we find additional cycles with more charge states involved. The AR $(m=2$, $q=2$ ) allows to create $n \Rightarrow n \pm 2 \Rightarrow n \pm 1 \Rightarrow n$ by having an AR through one, followed by two quasiparticle tunnelling processes through the other junction. In analogy to the J-QP cycle, this is called the A-QP cycle 10. Including MARs lead to cycles involving even more charge states like the MAR-QP cycle $n \Rightarrow n \pm 3 \Rightarrow n \pm 2 \Rightarrow n \pm 1 \Rightarrow n$ consisting of MAR $(m=3, q=2)$ and three quasiparticle tunnelling events. Combining AR and J-e on opposite junctions we find another $n \Rightarrow n \pm 2 \Rightarrow n \pm 1 \Rightarrow n$ cycle known as 3e-AR [10]. Similar to the J-e transport there is the possibility of combining a Josephson transport with the dissipative AR on opposite junction. Leaving no excess charge, this combination will cause the J-AR cycle.

Multi-charge tunneling over both junctions or cotunneling as described in Ref. 4] lead to features located at very low bias voltages, which do not follow the relation of eq. 1. However, with the quite small Josephson coupling of the $\mathrm{TB}$, these processes become very unlikely and their signatures vanish in the noise floor.

\subsection{Current Simulations}

We use Orthodox theory [3, 1, 25] to simulate the current through the SSET. In the regime of small coupling and large bias value, the discrete charge states of the island are sufficient to simulate the current using a master equation approach. We derive the bias and gate dependent change of the island's free energy $\delta$ for each transport process following the capacitive model (cf. eq. (1)). Using $\delta$ and the superconducting density of quasiparticle states [25] we simulate the quasiparticle rate $\Gamma_{\mathrm{QP}}(\delta)$. Additionally $\Gamma_{\mathrm{QP}}$ depends on the junction resistances and the temperature of the system. The resonant Josephson transport is harder to implement, since tunnelling of a Cooper pair is a nondissipative process. In Refs. 26, 27] a rate for Josephson transport linked to quasiparticle transport (useable for J-e) was derived

$$
\Gamma_{\mathrm{J}}(\delta)=\frac{\Gamma_{\mathrm{QP}} E_{\mathrm{J}}}{4 \delta^{2}+\left(h \Gamma_{\mathrm{QP}}\right)^{2}}
$$

depending on the Josephson coupling $E_{\mathrm{J}}$. This approach is known to describe the J-QP current feature quite well [28, but will not include correlated Josephson and Andreev transport. In our simulation $E_{\mathrm{J}}$ is calculated using the normal state resistance of the junction 9 .

The other dissipative processes, AR and MAR, are integrated in the simulation by using rates derived from the quasiparticle rate

$$
\Gamma_{\mathrm{AR}}(\delta)=G_{\mathrm{AR}} \Gamma_{\mathrm{QP}}(2 \delta), \quad \Gamma_{\mathrm{MAR}}(\delta)=G_{\mathrm{MAR}} \Gamma_{\mathrm{QP}}(3 \delta)
$$


by introducing conductances $G_{\mathrm{AR}}$ and $G_{\mathrm{MAR}}$. This method of implementing multiple electron tunnelling processes into the Orthodox theory is known to work in simulations for normal conducting-superconducting-normal conducting (NSN) SETs 29, 30, 18. At least for small $\tau$, this method of adding (M)AR is expected to qualitatively describe the phenomena correctly [31. Since the bias current relation for (M)AR deviates from the assumed step-like behaviour for higher $\tau$ [32], the simulation may present the related current features at underestimated bias.

\subsection{Tunnel Contact Regime}

Tuning the BJ to form a mere tunnel contact $\left(R_{\mathrm{BJ}}=825 \mathrm{k} \Omega\right)$, we find a classical SET behaviour. In the normal conducting state (Fig. 2), the low bias current is suppressed and a clear diamond pattern in the bias/gate-voltage plane is visible. Due to the highly asymmetric junctions $\left(R_{\mathrm{TB}}=112 \mathrm{k} \Omega\right)$, there is a faint hint of a diamond of second order.

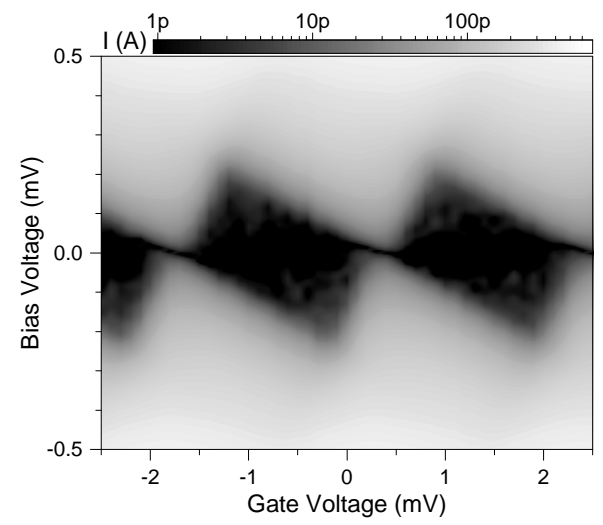

Fig. 2 Normal conducting bias-gatecurrent map with $R_{\mathrm{BJ}}=825 \mathrm{k} \Omega$ and $R_{\mathrm{TB}}=112 \mathrm{k} \Omega$. The current in the normal-conducting state shows the expected diamond shaped Coulomb blockade in low bias regime.

When switching to the superconducting state as seen in Figure 3 , the interplay of superconductivity and Coulomb blockade causes a variety of current cycles. As mentioned before, the transport diagram ${ }^{1}$ and some general considerations enable us to identify these cycles. If Josephson transport is involved features appear as peaks in the current, whereas dissipative processes lead to an onset. We label the lines with the initial and final charge state of the transport process $(n \Rightarrow n \pm m)$. Using the labels we analyse in which region of bias and gate voltage all individual transport processes required for a cycle are possible. Because the lines are graphical solutions of eq. (1), this is equivalent to the estimation of the energetic margins for each cycle as illustrated in Ref. [10].

\footnotetext{
1 The used parameters are $E_{\mathrm{C}}=122 \mu \mathrm{eV}, \delta C / C_{\Sigma}=-0.557, C_{G}=75 \mathrm{aF}, \Delta=190 \mu \mathrm{eV}$, $Q_{0} / e=0.625$
} 


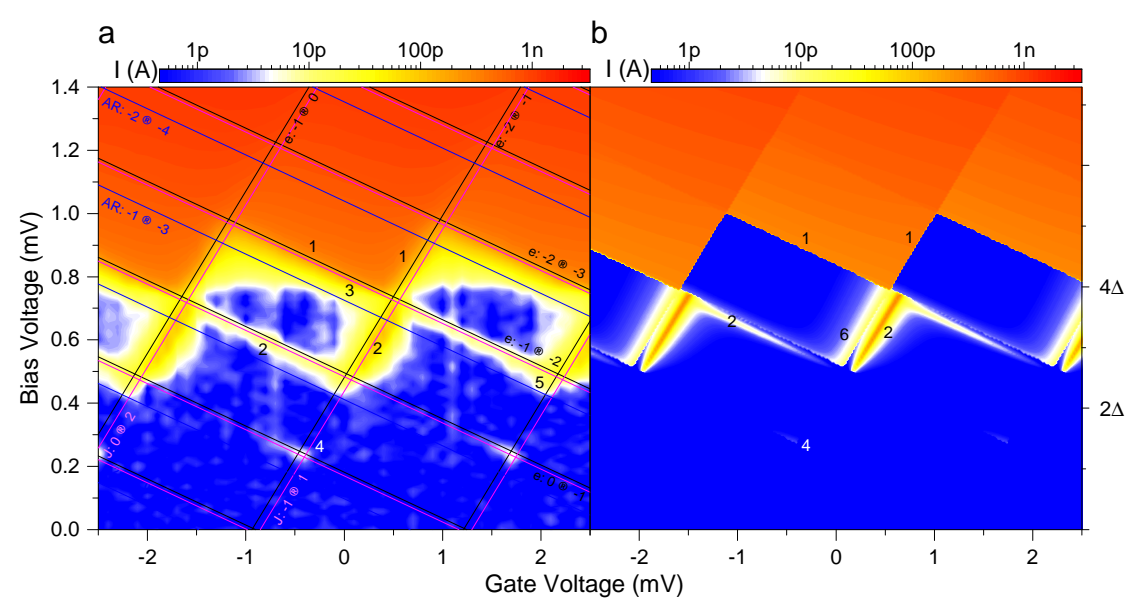

Fig. 3 Superconducting bias-gate-current map (a) with $R_{\mathrm{BJ}}=825 \mathrm{k} \Omega$ and $R_{\mathrm{TB}}=112 \mathrm{k} \Omega$. The current map is overlaid with the transport diagram (parameters in footnote 1 ) showing the onset condition for quasiparticle tunnelling (black) and the resonance condition for Cooper pair tunnelling (magenta) for both junctions. The onset condition for AR (blue) is shown for the BJ only. We identify current contributions carried by e-e (1), J-QP (2), A-QP (3), 3e (4) and 3e-A (5) cycles. The simulated current (b), which for clarity includes quasiparticle and Josephson transport only, reflects Josephson and quasiparticle related transport features. Colour online.

Thus, the ridge of the e-e onset (label 1) follows the quasiparticle lines of the transport diagram for $V_{\mathrm{B}} \geq 4 \Delta$. It starts at intersections of quasiparticle lines connecting the same charge states (e.g. e: $0 \Rightarrow 1$ and e: $1 \Rightarrow 0$ ). The peaklike J-QP features accordingly (label 2) follow the Josephson lines. The J-QP feature is in general enclosed in two of the quasiparticle lines (e.g. J: $0 \Rightarrow-2$ in e: $-1 \Rightarrow 0$ and e: $0 \Rightarrow 1)$. The lower line $(\mathrm{e}:-1 \Rightarrow 0)$ is the threshold for this specific J-QP cycle to be completed, the upper one (e: $0 \Rightarrow 1$ ) allows an additional charge to tunnel onto the island and destroys the resonance condition required for the Josephson transport. The absolute current of the JQP having the Josephson transport either over the BJ (negative slope) or the TB (positive slope) is different, which is caused by the different resistances. The small current preceding the e-e onset (label 3) is an A-QP cycle. The onset follows the Andreev line through the BJ (AR: $0 \Rightarrow-2$ ) and starts at the quasiparticle line $(\mathrm{e}:-1 \Rightarrow 0)$ required to complete the cycle. Since AR is a dissipative transport, the A-QP is not completely suppressed when reaching the next quasiparticle line (e: $0 \Rightarrow 1$ ). Very small signatures of $3 \mathrm{e}$ (label 4$)$ and 3e-AR (label 5) cycles are visible as well and are likewise identified by their shape and location.

For comparison we present the simulation in Fig $3 \mathrm{~b}$, which includes quasiparticle and Josephson transport only. The parameters used were determined approximating the transport diagram in the superconducting map and the normal state resistances of $R_{\mathrm{BJ}}=825 \mathrm{k} \Omega$ and $R_{\mathrm{TB}}=112 \mathrm{k} \Omega$. (M)AR pro- 
cesses are omitted in the simulation for the sake of clarity ${ }^{2}$ The simulation allows us to reproduce the prominent features in the SSET current, namely the e-e onset (label 1) and the J-QP cycle (label 2). The much smaller 3e current cycle (label 4 ) is also visible and comparable in height. An additional feature (label 5) is present in the simulation only and is an artefact of the rate equation approach and the linkage of the Josephson to the quasiparticle rates.

The J-QP cycle on the negative slope ( $\mathrm{J}$ over BJ) in the measurements is more pronounced than in the earlier realizations of SSETs consisting of two classical tunnel barriers of comparable resistance [4, 8, 10, 33]. A classical tunnel barrier having that high resistance should have negligible superconductive coupling, leading to a strong suppression of the J-QP cycle as seen in the simulation. Taking into account the BJ being nevertheless an atomic contact with small number of relatively high transmissive conduction channels explains the discrepancy of the estimated Josephson coupling and additionally the appearance of AR even in this high resistance regime. This property is a major difference to SSETs with classical tunnel barriers and one of the key physical results reported in this article. Further measurements on superconducting SETs are necessary to explore this behaviour in more detail.

\subsection{Near Tunnelling Regime}

When closing the BJ to some lower resistance value $\left(R_{\mathrm{BJ}}=73 \mathrm{k} \Omega\right)$ many new features appear. Please note that from this point on we present the conductance instead of the current. The normal conducting data (Fig. 4) shows the typical Coulomb diamonds. The conductance within the central diamond is completely suppressed and a diffuse diamond of second order appears. Even though the nominal resistances of both junctions are comparable $\left(R_{\mathrm{TB}}=112 \mathrm{k} \Omega\right)$, the BJ (negative slope) dominates the ridges in the conductance map suggesting very different contact properties.

This difference is much more pronounced in the superconducting data (Fig. 5a). Almost all sub-gap features appear on the negative slope and are therefore dominated by the different transport mechanisms over the BJ. In order to understand the features within OT, the transport-diagram ${ }^{3}$ featuring quasiparticle transport over both junctions plus Josephson and (M)AR transport over the BJ is drawn. Thereby we identify the e-e onset (label 1) and a very pronounced J-QP cycle (label 2). In contrast to the tunnel regime described above, the A-QP cycle (label 3) appears as a step-like onset in the current (a peak in conductance). The very different height of the e-e onset on the positive and negative slopes suggests having another cycle included in the second step-like onset on the negative slope. Respecting the MAR lines and

\footnotetext{
2 Including (M)AR in the master equation simulation of this regime even with very low conductance will cause numerical artefacts affecting the height and width of the J-QP cycle over the TB.

3 The used parameters are $E_{\mathrm{C}}=123 \mu \mathrm{eV}, \delta C / C_{\Sigma}=-0.557, C_{G}=74 \mathrm{aF}, \Delta=190 \mu \mathrm{eV}$, $Q_{0}=0.425$
} 


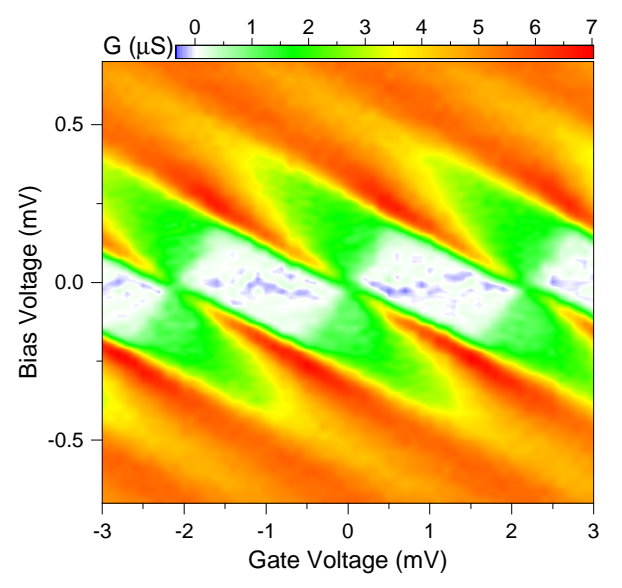

Fig. 4 Normalconducting bias-gateconductance map with $R_{\mathrm{BJ}}=72 \mathrm{k} \Omega$ and $R_{\mathrm{TB}}=112 \mathrm{k} \Omega$. The normal-conducting map shows the characteristic Coulomb blockade pattern with a diffused onset on the slope of the TB (positive). Colour online.

the corresponding labels the difference in height is caused by a MAR-QP cycle (e.g. MAR: $0 \Rightarrow-3$ in between e: $-1 \Rightarrow 0$ and e: $0 \Rightarrow 1$ ).

The very small 3e-A cycle (label 5 ) is barely visible, since this cycle includes the less pronounced Josephson transport over TB. This is also the reason why the J-QP feature with the Josephson transport over the TB is not visible. The feature at very low bias (label 6) could be caused by two different cycles, namely off resonance 3e [31] or A-J. An unambiguous identification is not possible due to missing bias and current resolution. Furthermore an unidentified feature (label ?) appears in the data. While its peak-like shape in the current is always observable, the position and extension of these features depend on the polarity of the bias.

One might wonder if the unknown feature is caused by charge fluctuators found in back gated SETs on silicon oxide, glass and sapphire substrates 34. Yet charge fluctuators would not explain the repetition of the feature in all diamonds and the observed asymmetry with respect to bias. In general, we rarely observe charge fluctuators in our samples, which might be a consequence of polyimide used as substrate.

The unidentified feature also appears in other contacts on this and other samples whenever MAR can be proven by the appearance of the MAR-QP cycle. With the current model of consecutive transport we do not find a cycle which explains this feature, satisfies all energetic requirements, and gives an explanation for the bias dependence. Nevertheless we believe the feature arises from some transport process depending on or involving MAR.

The simulation for $R_{\mathrm{BJ}}=73 \mathrm{k} \Omega$ from Fig. $5 \mathrm{~b}$ was carried out including the quasiparticle and Josephson transport through both junctions and enabling $\mathrm{AR}$ and MAR through the BJ. While the simulation is able to represent the occurrence and location of most of the distinct feature, the overall height of the features is not represented well at all. Due to the very sharp edges in the simulation, the numerical derivation overestimates all steps and peaks in current. Nevertheless the locations of the quasiparticle onset in combination 


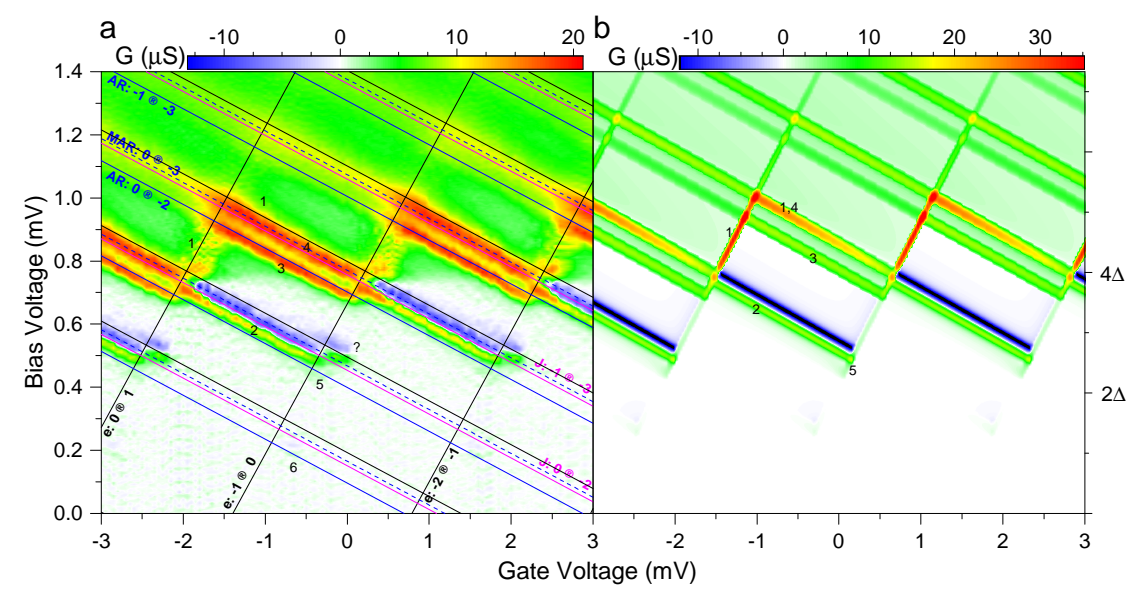

Fig. 5 Superconducting bias-gate-conductance map (a) with $R_{\mathrm{BJ}}=72 \mathrm{k} \Omega$ and $R_{\mathrm{TB}}=$ $112 \mathrm{k} \Omega$. There are many sub-gap features, which mostly appear due to (M)AR transport through the BJ (negative slope). The transport diagram (parameter in footnote 3 shown includes quasiparticle transport (black) through both TB and BJ as well as Josephson (magenta), AR (blue) and three charge MAR (blue dashed) for the BJ. The features can be identified as e-e (1), J-QP(2), A-QP(3), MAR-QP(4) and very small 3e-A(5) cycles. Another prominent feature (labelled as ?) is not covered by the current interpretation using OT. In the deep subgap region there are indications for 3e and J-A (6) processes very close to each other. All identified features also appear in the simulation (b), which considers quasiparticle, Josephson, AR and MAR transport. The difference in their magnitude is caused by the very sharp edges of the simulation and the numerical derivation. The simulation does not cover the unknown feature (?) either. Colour online.

with the MAR-QP cycle (label 1,4), the J-QP cycle (label 2), the A-QP (label 3 ) and the 3e-A cycle (label 5) are reflected quite well. The fact that there is no 3e cycle within the gap and the simulation neglects J-AR transport suggests that the feature observed in the measurement (Fig. 5a, label 6) is caused by J-AR cycles. As expected, the unidentified feature in the experimental data (Fig. 5 a, label ?), does not appear in the simulations.

\subsection{Point Contact Regime}

The BJ can be adjusted to form an atomic point contact. This is achieved during opening the junction by stopping the mechanical drive at the latest conductance plateau before breaking [20]. The normal state conductance of the SET with $R_{\mathrm{BJ}}=10 \mathrm{k} \Omega$ differs from the previously shown data in the reduction of the Coulomb blockade effect as seen in Fig. 6a. The low bias conductance is never completely suppressed. Furthermore no clear diamond pattern of the $\mathrm{CB}$ is visible. In this regime it is quite difficult to extract the required set of parameters of the SET from the normal conducting data.

The conductance of the superconducting state (Fig. 6b) also features no clear signs of Coulomb blockade as expected from OT. No e-e onset at $V_{\mathrm{B}} \geq$ 

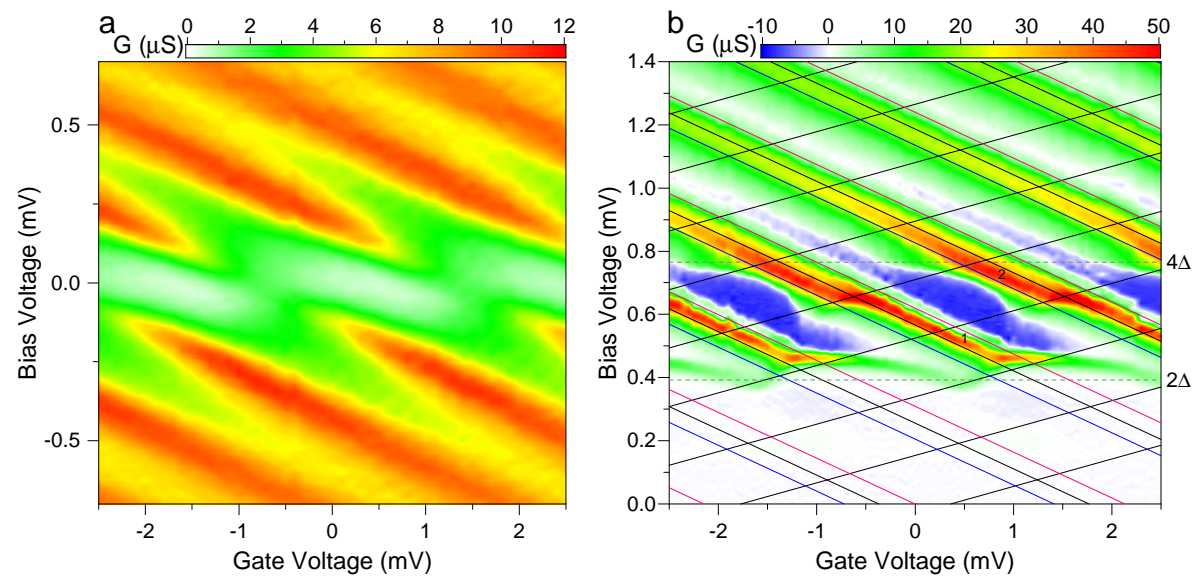

Fig. 6 Normal (a) and superconducting (b) bias-gate-conductance map with $R_{\mathrm{BJ}}=10 \mathrm{k} \Omega$ and $R_{\mathrm{TB}}=112 \mathrm{k} \Omega$. In the normal conducting state (a) the Coulomb blockade does not completely suppress the low bias conductance. The typical diamond shape is blurred. Transport over the BJ (negative slope) dominates the measurement. The superconducting measurement (b) is equally dominated by transport over the BJ. The transport diagram (parameters in footnote 4) was drawn utilizing the assignment of J-QP (1) and A-QP (2) cycles as described in the text and includes lines for quasiparticle (black), Josephson (magenta) and AR (blue) transport. Instead of a quasiparticle onset a faintly modulated onset slightly above $2 \Delta$ is observed. The transport diagram points to a distinctive reduction of $E_{\mathrm{C}}$. Colour online.

$4 \Delta / e$ is visible, instead a slightly gate modulated onset at $V_{\mathrm{B}} \geq 2 \Delta / e$ appears. This onset does not follow the edges of a distinct diamond pattern but resembles a quasiparticle onset. However, the pronounced ridges in the conductance data can be related to well-known cycles. We suppose the highest contribution through the BJ in the atomic contact regime to be either Josephson transport or AR. Inspired by the OT, we tentatively assign the peak-valley ridge (peak in current) to be caused by the J-QP cycle (label 1). Accordingly the second, peak-like feature is identified as A-QP (label 2). This allows us to pinpoint the complete transport diagram ${ }_{4}^{4}$ including the lines for quasiparticle, Josephson and AR transport 5 . These lines match well with characteristic changes in the conductance map. Nevertheless, not all observations can be described within this model. For example, following OT, the J-QP cycle should be suppressed once the limiting quasiparticle threshold $\left(2 \Delta+3 E_{\mathrm{C}}\right)$ is passed. However, we observe that the process persists to higher bias values $(\approx 4 \Delta)$. We argue that in the strong coupling regime realized here the OT is not applicable anymore and thus no simulations were performed.

\footnotetext{
4 The used parameters are $E_{\mathrm{C}}=58 \mu \mathrm{eV}, \delta C C_{\Sigma}=0.252, C_{G}=75 \mathrm{aF}, \Delta=190 \mu \mathrm{eV}$, $Q_{0} / e=0.002$

5 The AR line is not on top of the ridge intentionally. We expect the maximum in the Andreev conduction to be not at the threshold but at slightly higher bias values for high transmissions as seen in point contacts 32. The harder criterion is in our opinion the beginning of the J-QP cycle at the intersection to the quasiparticle line.
} 
4.6 Renormalization of $E_{\mathrm{C}}$

Although the OT does not quantitatively describe the transport through the SSET in the point contact regime, it allows extracting general parameters such as the charging energy. We used measurements in the normal conducting state on the same sample, but varying in $R_{\mathrm{BJ}}$, as basis for the data provided in Fig. 7 . We verified that these $E_{\mathrm{C}}$ values agree with the ones in the superconducting state within the given error bars. Measurements with obvious rearrangement in the BJ during the mapping were excluded. We find that once the point contact regime is approached, i.e. $R_{\mathrm{BJ}} \leq 1 / G_{0} \simeq 12.9 \mathrm{k} \Omega$, the charging energy of the SET reduces drastically from roughly $115 \mu \mathrm{eV}$ to about $86 \mu \mathrm{eV}$.

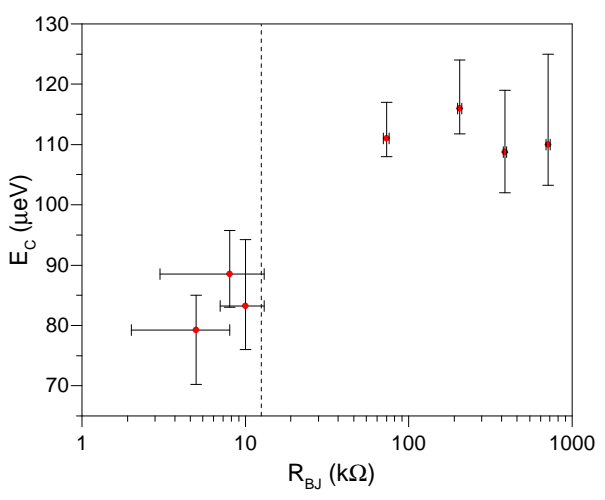

Fig. 7 The behaviour of $E_{\mathrm{C}}$ vs. the BJ resistance, extracted from several different contacts in normal conducting state and verified in the superconducting state, shows a clear reduction of $E_{\mathrm{C}}$ once the BJ forms a point contact $\left(R_{\mathrm{BJ}} \leq 1 / G_{0}\right.$, indicated $)$. The error bars indicate the minimal and maximal values of $E_{\mathrm{C}}$ for which the experimental transport diagram is in agreement with the measurements. Likewise the uncertainty for the resistance arises from the minimal and maximal resistance, obtained by linear fitting the IV characteristics at high bias.

Based on the data we cannot reveal whether the reduction occurs suddenly or as a continuous decrease. The missing range of resistances is difficult to address using the break junction technique due to the limited mechanical stability in the close tunnelling regime. Renormalization of $E_{\mathrm{C}}$ has been observed before in SETs of two dimensional electron gases [35, 36] as well as in $\mathrm{Al} / \mathrm{AlO}_{\mathrm{x}} / \mathrm{Al}$ structures with two oxide barriers [31, 37. In these works, however, the $E_{\mathrm{C}}$ values of different samples had to be compared. Additionally the recent work of Ref. [7] showed the disappearance of the zero bias Coulomb oscillations with increasing conductance. Nevertheless, a change in $E_{\mathrm{C}}$ was not reported. From the theoretical side a reduction of $E_{\mathrm{C}}$ when increasing the coupling is expected for macroscopic systems [37, 38] as well as for contacts with single transmission channels [39, 40]. Nazarov [41] predicted an exponential reduction of $E_{\mathrm{C}}$ depending on the nature of the tunnel contacts and conductance. Albeit a change in the junction capacitance when closing the BJ cannot be excluded as a source of change in $E_{\mathrm{C}}$. Further measurements are necessary to explore this observation in more detail. 


\section{Summary}

In conclusion, we present a testbed system for systematic studies of the Coulomb blockade and superconducting transport covering the whole range from the weakly to the strongly coupled regime within one sample. This is realized by using one mechanically controllable break junction and an oxide tunnel barrier. In the weak tunnelling regime the transport behaviour in both the normal and the superconducting state is well described by the Orthodox theory. When forming a near tunnelling contact in the superconducting state, we observe novel current features, some being related to multiple Andreev reflection, some of unknown origin, that are not reported in theory so far. Approaching the point contact regime the Coulomb blockade behaviour is washed out and we find a renormalization of the charging energy toward smaller values. Additional studies are necessary to clarify how the charging energy vanishes.

Acknowledgements The authors like to thank Jens Siewert and Wolfgang Belzig for useful discussion. We also thank Olivier Schecker, Ursula Schröter, and Paramita Kar Choudhury for their contributions in the early state of the project.

\section{References}

1. K. Likharev, IEEE Trans. Magn. 23, 1142 (1987). DOI 10.1109/TMAG.1987.1065001

2. D.V. Averin, K.K. Likharev, J. Low Temp. Phys. 62, 345 (1986). DOI 10.1007/BF00683469

3. T.A. Fulton, G.J. Dolan, Phys. Rev. Lett. 59, 109 (1987). DOI 10.1103/PhysRevLett.59.109

4. P. Joyez, The single cooper pair transistor: A macroscopic quantum system. PhD Thesis, Université Pierre et Marie Curie-Paris VI (1995)

5. D. Chouvaev, L.S. Kuzmin, D.S. Golubev, A.D. Zaikin, Phys. Rev. B 59, 10599 (1999). DOI 10.1103/PhysRevB.59.10599

6. C. Wallisser, B. Limbach, P. vom Stein, R. Schäfer, in International Workshop on Superconducting Nano-Electronics Devices, ed. by J. Pekola, B. Ruggiero, P. Silvestrini (Springer, Boston, MA, 2002), pp. 123-132. DOI 10.1007/978-1-4615-0737-6_14

7. S. Jezouin, Z. Iftikhar, A. Anthore, F.D. Parmentier, U. Gennser, A. Cavanna, A. Ouerghi, I.P. Levkivskyi, E. Idrisov, E.V. Sukhorukov, L.I. Glazman, F. Pierre, Nature 536, 58 (2016). DOI 10.1038/nature19072

8. T.A. Fulton, P.L. Gammel, D.J. Bishop, L.N. Dunkleberger, G.J. Dolan, Phys. Rev. Lett. 63, 1307 (1989). DOI 10.1103/PhysRevLett.63.1307

9. P. Hadley, E. Delvigne, E.H. Visscher, S. Lähteenmäki, J.E. Mooij, Phys. Rev. B 58, 15317 (1998). DOI 10.1103/PhysRevB.58.15317

10. R.J. Fitzgerald, S.L. Pohlen, M. Tinkham, Phys. Rev. B 57, R11073 (1998). DOI 10.1103/PhysRevB.57.R11073

11. T.M. Klapwijk, G.E. Blonder, M. Tinkham, Phys. B 109, 1657 (1982) 
12. M. Octavio, M. Tinkham, G.E. Blonder, T.M. Klapwijk, Phys. Rev. B 27, 6739 (1983). DOI 10.1103/PhysRevB.27.6739

13. Y. Avishai, A. Golub, A.D. Zaikin, Europhys. Lett. 54, 640 (2001). DOI 10.1209/epl/i2001-00340-1

14. T.M. Eiles, J.M. Martinis, M.H. Devoret, Phys. Rev. Lett. 70, 1862 (1993). DOI 10.1103/PhysRevLett.70.1862

15. F.W.J. Hekking, L.I. Glazman, K.A. Matveev, R.I. Shekhter, Phys. Rev. Lett. 70, 4138 (1993). DOI 10.1103/PhysRevLett.70.4138

16. M.R. Buitelaar, W. Belzig, T. Nussbaumer, B. Babić, C. Bruder, C. Schönenberger, Phys. Rev. Lett. 91, 057005 (2003). DOI 10.1103/PhysRevLett.91.057005

17. J.C. Cuevas, A. Martín-Rodero, A. Levy Yeyati, Phys. Rev. B 54, 7366 (1996). DOI 10.1103/PhysRevB.54.7366

18. V.F. Maisi, O.P. Saira, Y.A. Pashkin, J.S. Tsai, D.V. Averin, J.P. Pekola, Phys. Rev. Lett. 106, 217003 (2011). DOI 10.1103/PhysRevLett.106.217003

19. T. Greibe, M.P.V. Stenberg, C.M. Wilson, T. Bauch, V.S. Shumeiko, P. Delsing, Phys. Rev. Lett. 106, 097001 (2011). DOI 10.1103/PhysRevLett.106.097001

20. E. Scheer, P. Joyez, D. Esteve, C. Urbina, M.H. Devoret, Phys. Rev. Lett. 78, 3535 (1997). DOI 10.1103/PhysRevLett.78.3535

21. C. Schirm, M. Matt, F. Pauly, J.C. Cuevas, P. Nielaba, E. Scheer, Nat. Nanotechnol. 8, 645 (2013). DOI 10.1038/nnano.2013.170

22. A.I. Yanson, J.M. van Ruitenbeek, Phys. Rev. Lett. 79, 2157 (1997). DOI 10.1103/PhysRevLett.79.2157

23. M. Thalmann, T. Pietsch, H.F. Pernau, C. Strunk, E. Scheer, Comparison of cryogenic low-pass filters. , Rev. Sci. Instrum. (in press)

24. Y. Nakamura, C.D. Chen, J.S. Tsai, Phys. Rev. B 53, 8234 (1996). DOI 10.1103/PhysRevB.53.8234

25. G. Schön, in Quantum Transport and Dissipation, ed. by T. Dittrich, 1st edn. (Wiley-VCH, Weinheim ; New York, 1998), p. 149

26. V.Y. Aleshkini, D.V. Averin, Phys. B 165, 949 (1990). DOI 10.1016/S0921-4526(09)80060-4

27. D. Averin, V.Y. Aleshkin, JETP Lett. 50, 9 (1989)

28. S.L. Pohlen, R.J. Fitzgerald, M. Tinkham, Phys. B 284, 1812 (2000). DOI 10.1016/S0921-4526(99)03027-6

29. J. Siewert, Europhys. Lett. 46, 768 (1999). DOI 10.1209/epl/i1999-001214

30. S. Rajauria, P. Gandit, T. Fournier, F.W.J. Hekking, B. Pannetier, H. Courtois, Phys. Rev. Lett. 100, 207002 (2008). DOI 10.1103/PhysRevLett.100.207002

31. S.L. Pohlen, The superconducting single-electron transistor. PhD Thesis, Harvard University (1999)

32. J.C. Cuevas, W. Belzig, Phys. Rev. Lett. 91, 187001 (2003). DOI 10.1103/PhysRevLett.91.187001 
33. J.M. Hergenrother, M.T. Tuominen, T.S. Tighe, M. Tinkham, IEEE Trans. Appl. Supercond. 3, 1980 (1993). DOI 10.1109/77.233570

34. A. Pourkabirian, V. Gustafsson, M. G. Johansson, J. Clarke, P. Delsing, Phys. Rev. Lett. 113, 256801 (2014). DOI 10.1103/PhysRevLett.113.256801

35. E.B. Foxman, P.L. McEuen, U. Meirav, N.S. Wingreen, Y. Meir, P.A. Belk, N.R. Belk, M.A. Kastner, S.J. Wind, Phys. Rev. B 47, 10020 (1993). DOI 10.1103/PhysRevB.47.10020

36. L.W. Molenkamp, K. Flensberg, M. Kemerink, Phys. Rev. Lett. 75, 4282 (1995). DOI 10.1103/PhysRevLett.75.4282

37. D.V. Averin, A.N. Korotkov, A.J. Manninen, J.P. Pekola, Phys. Rev. Lett. 78, 4821 (1997). DOI 10.1103/PhysRevLett.78.4821

38. G. Falci, G. Schön, G.T. Zimanyi, Phys. Rev. Lett. 74, 3257 (1995). DOI 10.1103/PhysRevLett.74.3257

39. K. Flensberg, Phys. Rev. B 48, 11156 (1993). DOI 10.1103/PhysRevB.48.11156

40. K. Flensberg, Phys. B 203, 432 (1994). DOI 10.1016/0921-4526(94)900922

41. Y.V. Nazarov, Phys. Rev. Lett. 82, 1245 (1999). DOI 10.1103/PhysRevLett.82.1245 